\title{
On the quantification of entanglement in infinite-dimensional quantum systems
}

\author{
Jens Eisert ${ }^{1}$, Christoph Simon ${ }^{2}$, and Martin B. Plenio ${ }^{1}$ \\ 1 QOLS, Imperial College of Science, Technology and Medicine, London, SW7 2BW, UK \\ 2 Clarendon Laboratory, University of Oxford, Oxford OX1 3PU, UK
}

\begin{abstract}
We investigate entanglement measures in the infinite-dimensional regime. First, we discuss the peculiarities that may occur if the Hilbert space of a bi-partite system is infinitedimensional, most notably the fact that the set of states with infinite entropy of entanglement is trace-norm dense in state space, implying that in any neighbourhood of every product state lies an arbitrarily strongly entangled state. The starting point for a clarification of this counterintuitive property is the observation that if one imposes the natural and physically reasonable constraint that the mean energy is bounded from above, then the entropy of entanglement becomes a trace-norm continuous functional. The considerations will then be extended to the asymptotic limit, and we will prove some asymptotic continuity properties. We proceed by investigating the entanglement of formation and the relative entropy of entanglement in the infinite-dimensional setting. Finally, we show that the set of entangled states is still tracenorm dense in state space, even under the constraint of a finite mean energy.
\end{abstract}

PACS-numbers: 03.67.-a, 03.65.Bz

\section{INTRODUCTION}

When A. Einstein, B. Podolski, and N. Rosen published their seminal paper on the question whether the quantum mechanical description of physical reality could be considered complete, they formulated their gedanken experiment in terms of two quantum systems that are entangled through their position and momentum. In the well-known reformulation by $\mathrm{D}$. Bohm the entangled quantum systems are replaced by two spin-1/2-particles, each of which is supplemented with a two-dimensional Hilbert space [1]. In this formulation the physical situation can be discussed without the need to take the technicalities of infinite-dimensional spaces into account. Most of the theory of quantum information has in fact been developed for finitedimensional settings, the qubit - the two-level system - being the prototypical elementary quantum system in this context [2]. Only quite recently, several contributions in the field addressed issues of quantum information in infinite-dimensional settings [3-7]. Experiments have been performed with quantum optical systems where not the polarisation degrees of freedom have been employed, but the ones corresponding to the canonical coordinates of the field modes. Influenced by such experiments the theory of entanglement has been extended to systems with infinitedimensional Hilbert spaces, most notably for so-called Gaussian quantum states, which form an important class of states from a practical point of view [䧋]. In 
particular, the question of separability and distillability of Gaussian states of multimode systems is essentially solved. Concerning the theory of entanglement of nonGaussian states, however, not so much is known to date.

It is the purpose of this paper to comment on some peculiarities that may occur in the infinite-dimensional setting when quantifying the degree of entanglement, and on ways to retain meaningful measures of entanglement. The starting point is the following observation: on a bi-partite infinite-dimensional Hilbert space there always exist pure states which are arbitrarily close to a pure product state - as quantified by the trace-norm difference - but they exhibit an entropy of entanglement which is infinite. In this sense the assignment of the value zero of the entanglement of the pure product state is not entirely unambigous. A similar situation can occur in the mixed-state domain. The key insight leading to a possible resolution of this problem is derived from an investigation of the mean energy of the involved quantum states: If one imposes the natural requirement that the mean energy of the involved states is bounded from above, then one retains meaningful measures of entanglement. In other words, although there are indeed sequences of pure states that converge to a certain pure state in trace-norm while the entropy of entanglement is divergent, their energy must necessarily diverge.

The scope of this paper is quite modest: We will neither characterize entanglement measures through certain natural axioms, nor try to equip the known entanglement measures with a clearcut operational interpretation in the infinite-dimensional setting. Instead, this paper presents a collection of clarifying statements concerning the continuity of entanglement measures. We will discuss the trace-norm continuity of the entropy of entanglement and an asymptotic continuity property. In addition, we will study the relative entropy of entanglement and the entanglement of formation. In the last section we will consider the question of the existence of a separable neighbourhood of some mixed state under a constraint on the mean energy.

\section{NOTATION}

We start by clarifying the notation that will be used in this paper. We will consider the situation where the composite system consists of two parts $A$ and $B$ (each of which having finitely many degrees of freedom) such that the Hilbert space of the joint system can be written as $\mathcal{H}=\mathcal{H}_{A} \otimes \mathcal{H}_{B}$. Throughout the paper we will assume that $\operatorname{dim}\left[\mathcal{H}_{A}\right]=\infty$ and $\operatorname{dim}\left[\mathcal{H}_{B}\right]=\infty$. The notation $\mathcal{S}(\mathcal{H})$ will be used for the set of density operators on $\mathcal{H}$, that is, the set of positive trace-class operators with trace one. In the same way the set $\mathcal{S}\left(\mathcal{H}_{A}\right)$ is defined as the state space on $\mathcal{H}_{A}$. The symbol $\|$.$\| will be reserved for the trace-norm, which is defined as \|A\|=\operatorname{tr}[|A|]=$ $\operatorname{tr}\left[\left(A^{\dagger} A\right)^{1 / 2}\right]$ for operators $A$, while the standard operator norm will be written as ||l.|l. For our purposes it will also be necessary to impose a certain requirement on the Hamiltonian $H=H_{A} \otimes 1+1 \otimes H_{B}$ of the bi-partite system. It will be demanded that $H$ has a discrete spectrum, such that the spectral decomposition of $H_{A}$ and $H_{B}$ reads as

$$
H_{A}=\sum_{n=0}^{\infty} \epsilon_{A}^{(n)} \pi_{A}^{(n)} \text { and } H_{B}=\sum_{n=0}^{\infty} \epsilon_{B}^{(n)} \pi_{B}^{(n)},
$$


respectively, where $\epsilon_{A}^{(i)} \leq \epsilon_{A}^{(j)}$ and $\epsilon_{B}^{(i)} \leq \epsilon_{B}^{(j)}$ for $i<j . \pi_{A}^{(n)}$ are the projectors on the one-dimensional spaces spanned by the mutually orthogonal $\phi_{A}^{(n)} \in \mathcal{H}_{A}$, and analogously for $\pi_{B}^{(n)}$. We will always assume that the Hamiltonian $H$ of the system has the property that

$$
\operatorname{tr}\left[e^{-\beta H}\right]<\infty
$$

for all $\beta>0$. This is a natural assumption: it is simply required that the Gibbs state exists for all inverse temperatures of the system. In particular, it means that there can be no limit points in the spectrum of $H$, which in turn implies that no eigenvalue can be infinitely degenerate.

\section{ENTROPY OF ENTANGLEMENT}

Entanglement measures give an account of the degree of entanglement of a given quantum state. They are functionals mapping state space on the set of non-negative real numbers; the larger the number is, the more entangled is the quantum state. For pure states we will investigate the entropy of entanglement $E: \mathcal{S}(\mathcal{H}) \longrightarrow \mathbb{R}^{+}$(or reduced entropy), defined as

$$
E(\rho):=\left(S \circ \Phi_{B}\right)(\rho) .
$$

Here, $S: \mathcal{S}\left(\mathcal{H}_{A}\right) \longrightarrow \mathbb{R}^{+}$denotes the von-Neumann entropy, which is defined as $S(\rho)=-\operatorname{tr}\left[\rho \log _{2}(\rho)\right]$, and $\Phi_{B}: \mathcal{S}(\mathcal{H}) \longrightarrow \mathcal{S}\left(\mathcal{H}_{A}\right)$ is the partial trace with respect to system $B$. The entropy of entanglement quantifies to what extent the state departs from a product state. As has already been pointed out in the introduction, the entropy of entanglement may be very different from zero for states which are very 'close' to pure product states:

Example 1. Let $\sigma_{0}=\left|\psi_{0}\right\rangle\left\langle\psi_{0}\right|, \psi_{0}=\phi_{A}^{0} \otimes \phi_{B}^{0}$, be the ground state of the bi-partite system, and let $\left\{\sigma_{k}\right\}_{k=1}^{\infty}$ be a sequence of pure states $\sigma_{k}=\left|\psi_{k}\right\rangle\left\langle\psi_{k}\right|$, where

$$
\psi_{k}:=\left(1-\delta_{k}\right)^{1 / 2} \psi_{0}+\left(\delta_{k} / k\right)^{1 / 2} \sum_{n=1}^{k} \phi_{A}^{(n)} \otimes \phi_{B}^{(n)} .
$$

and $\delta_{k}:=1 / \log (k)$. In fact, the series $\left\{\sigma_{k}\right\}_{k=1}^{\infty}$ converges to $\sigma_{0}$ in trace-norm, i.e., $\lim _{k \rightarrow \infty}\left\|\sigma_{k}-\sigma_{0}\right\|=0$. However,

$$
\lim _{k \rightarrow \infty} E\left(\sigma_{k}\right)=1,
$$

whereas $E\left(\sigma_{0}\right)=0$, since $\sigma_{0}$ it is a product state.

That is to say, $E=S \circ \Phi_{B}$ is clearly not continuous in $\sigma_{0}$ : There are states which are arbitrarily close to the product ground state with respect to the trace-norm, but they have an entropy of entanglement that approaches a different value. As the trace-norm difference is an upper bound for differences in expectation values of all contractions, it is clear that the smaller the trace-norm difference of a pair of states is, 
the more difficult is it to distinguish the states by operational means. The situation is actually even more surprising, as the set of states which have infinite von-Neumann entropy is trace-norm dense in state space [8]. As can be easily verified, the set of pure states with infinite entropy of entanglement is also trace-norm dense in the set of all pure states:

Proposition 2. For all $\psi \in \mathcal{H}$ and all $\varepsilon>0$ there exist a vector $\phi \in \mathcal{H}$ such that $\||\psi\rangle\langle\psi|-| \phi\rangle\langle\phi| \|<\varepsilon$ and $\left(S \circ \Phi_{B}\right)(|\phi\rangle\langle\phi|)=\infty$.

Proof. According to the Schmidt decomposition (which can be applied in this infinite-dimensional setting) there exists an orthonormal basis $\left\{\psi_{A}^{(n)}\right\}_{n=1}^{\infty}$ of $\mathcal{H}_{A}$ and a basis $\left\{\psi_{B}^{(n)}\right\}_{n=1}^{\infty}$ of $\mathcal{H}_{B}$ such that $\psi \in \mathcal{H}$ can be written in the form

$$
\psi=\sum_{n=1}^{\infty}\left(p^{(n)}\right)^{1 / 2} \psi_{A}^{(n)} \otimes \psi_{B}^{(n)},
$$

where $\left\{p^{(n)}\right\}_{n=0}^{\infty}$ forms a probability distribution. For each $n \in \mathbb{N}$, define a sequence $\left\{q_{k}^{(n)}\right\}_{k=1}^{\infty}$ through $q_{k}^{(1)}=p_{k}$, and

$$
q_{k}^{(n)}:=\frac{p^{(n)}+1 /\left(k n \log _{2}(n)^{2}\right)}{\delta_{k}}, \quad n=2,3, \ldots,
$$

where $\delta_{k}>0$ is chosen in such a way that $\left\{q_{k}^{(n)}\right\}_{n=1}^{\infty}$ is also a probability distribution. This gives rise to a sequence of state vectors $\psi_{k} \in \mathcal{H}$ defined as

$$
\psi_{k}:=\sum_{n=1}^{\infty}\left(q_{k}^{(n)}\right)^{1 / 2} \psi_{A}^{(n)} \otimes \psi_{B}^{(n)} .
$$

The sequence $\left\{p^{(n)}\right\}_{n=0}^{\infty}$ is convergent; on using the fact that $f:[0,1] \longrightarrow \mathbb{R}^{+}$defined as $f(x)=-x \log _{2}(x)$ is monotone increasing in $[0, \varepsilon]$ for some $\varepsilon>0$, one can show that $\left(S \circ \Phi_{B}\right)\left(\left|\psi_{k}\right\rangle\left\langle\psi_{k}\right|\right)=\infty$ for all $k \in \mathbb{N}$. However,

$$
\lim _{k \rightarrow \infty} \||\psi\rangle\left\langle\psi|-| \psi_{k}\right\rangle\left\langle\psi_{k}\left|\|=\lim _{k \rightarrow \infty} \sum_{n=1}^{\infty}\right| p^{(n)}-q_{k}^{(n)}\right|=0 .
$$

The key observation in a possible clarification of this issue concerns the mean energy of the states. In the above example, the mean energy $\operatorname{tr}\left[\sigma_{k} H\right]$ grows beyond all bounds. After all, the energy that can possibly be invested in the preparation of a quantum state is in all instances limited. This is no accident, and we will see that in sequence of states $\left\{\sigma_{k}\right\}_{k=1}^{\infty}$ that converges in trace-norm to some state $\sigma$ such that the sequence of the entropies of entanglement of $\sigma_{k}$ does not converge to the entropy of entanglement of $\sigma$, the mean energy necessarily diverges.

Let $\mathcal{S}_{M}(\mathcal{H}) \subset \mathcal{S}(\mathcal{H})$ for a given number $M>0$ and for a given Hamiltonian $H$ be the set of states with a mean energy of at most $M$,

$$
\mathcal{S}_{M}(\mathcal{H}):=\{\rho \in \mathcal{S}(\mathcal{H}) \mid \operatorname{tr}[\rho H]<M\}
$$


Although this set is nowhere dense in state space - as $H$ is an unbounded operator according to the previous assumptions - it is a reasonable subset of the state space: it simply reflects the natural requirement that the mean energy is bounded from above. On this set, it turns out, several entanglement measures do not exhibit the above pathologies. We start with the trace-norm continuity of the entropy of entanglement, which is a corollary of a theorem in Ref. [8] due to A. Wehrl.

Proposition 3. Let $M>0$, let $\sigma \in \mathcal{S}_{M}(\mathcal{H})$, and let $\left\{\sigma_{k}\right\}_{k=1}^{\infty}$ be a sequence of states $\sigma_{k} \in \mathcal{S}_{M}(\mathcal{H})$ satisfying $\sigma_{k} \longrightarrow \sigma$ in trace-norm. Then

$$
\lim _{k \rightarrow \infty}\left|E(\sigma)-E\left(\sigma_{k}\right)\right|=0,
$$

that is, the entropy of entanglement $E: \mathcal{S}_{M}(\mathcal{H}) \longrightarrow \mathbb{R}^{+}$is a trace-norm continuous functional.

Proof. This statement is a consequence of a statement concerning the continuity of the von-Neumann entropy under an appropriate constraint of the energy [8]?]: If $\left\{\omega_{k}\right\}_{k=1}^{\infty}$ is a sequence of states taken from $\mathcal{S}_{M}(\mathcal{K})$ satisfying $\omega_{k} \rightarrow \omega$ in trace-norm for some state $\omega \in \mathcal{S}_{M}(\mathcal{K})$, together with the above assumptions concerning the spectrum of $H$, then

$$
\lim _{k \rightarrow \infty} S\left(\omega_{k}\right)=S(\omega)
$$

The states $\omega_{k}:=\Phi_{B}\left(\sigma_{k}\right)$ with $\mathcal{K}:=\mathcal{H}_{A}$ form such a sequence, since

$$
\left\|\Phi_{B}\left(\sigma_{k}\right)-\Phi_{B}(\sigma)\right\| \leq\left\|\sigma_{k}-\sigma\right\|
$$

for all $k \in \mathbb{N}$ by the contraction property of the trace-norm under completely positive maps, and since

$$
\operatorname{tr}\left[H_{A} \Phi_{B}\left(\sigma_{k}\right)\right] \leq \operatorname{tr}\left[H \sigma_{k}\right]<M .
$$

Hence, $\lim _{k \rightarrow \infty}\left|\left(S \circ \Phi_{B}\right)\left(\sigma_{k}\right)-\left(S \circ \Phi_{B}\right)(\sigma)\right|=0$ if $\lim _{k \rightarrow \infty}\left\|\sigma_{k}-\sigma\right\|=0$.

Therefore, if one introduces the natural and physically reasonable restriction that the mean energy must be bounded from above, then the entropy of entanglement becomes continuous, and a situation as in Example 1 or in Proposition 2 is excluded through this restriction [9]. Also, the entropy of entanglement is in all instances finite for pure states taken from $\mathcal{S}_{M}(\mathcal{H})$ for $M>0$. The statement of Proposition 3 alone does not, however, guarantee that the entropy of entanglement has appropriate continuity properties to describe entanglement manipulation in the asymptotic limit [10-14], in the sense of the limit of infinitely many identically prepared quantum systems. In particular, in order to give the entropy of entanglement an interpretation in terms of optimal achievable conversion rates in distillation-type protocols [10], one would aim at finding the following continuity property: Take a large number $m$ of identically prepared quantum systems in a state $\rho$. In a distillation procedure one would like to convert the state $\rho^{\otimes m}$ of these systems into a product 
state $\sigma^{\otimes n}$ consisting of a number of highly entangled states $\sigma$ of finite-dimensional quantum systems. For any finite number of identically prepared systems this procedure may not be possible in an optimal manner. One would then obtain a state $\sigma_{n}$, which is similar to yet not identical with $\sigma^{\otimes n}$. One may nevertheless require that the transformation is optimal in the asymptotic limit $n \longrightarrow \infty$. Roughly speaking, the measure of entanglement should then assign a value to $\sigma_{n}$ which is very similar to the value for $\sigma^{\otimes n}$, such that the difference per copy becomes negligible in the asymptotic limit. Note that none of the mixed states $\sigma_{n}$ are required to have a finitedimensional carrier. It is the purpose of the subsequent proposition to show that under an appropriate restriction on the mean energies, the entropy of entanglement exhibits an asymptotic continuity property [15].

Proposition 4. Let $\sigma \in \mathcal{S}_{M}(\mathcal{H}), M>0$, be a pure state that is supported on a finitedimensional subspace of $\mathcal{S}(\mathcal{H})$, and let $\left\{\sigma_{n}\right\}_{n=1}^{\infty}, \sigma_{n} \in \mathcal{S}_{n M}\left(\mathcal{H}^{\otimes n}\right)$, be a sequence of states satisfying

$$
\lim _{n \rightarrow \infty}\left\|\sigma_{n}-\sigma^{\otimes n}\right\|=0
$$

Then

$$
\lim _{n \rightarrow \infty} \frac{\left|E\left(\sigma^{\otimes n}\right)-E\left(\sigma_{n}\right)\right|}{n}=0 .
$$

The subsequent lemma will prepare the proof. The property of the von-Neumann entropy that will be investigated reminds of the lower semi-continuity property [17]. In our case, however, we consider a sequence of states which are defined on a sequence of Hilbert spaces of increasing dimension.

Lemma 5. Let $\omega$ be a state that is supported on a finite-dimensional subspace of $\mathcal{S}\left(\mathcal{H}_{A}\right)$, and let $\left\{\omega_{n}\right\}_{n=1}^{\infty}, \omega_{n} \in \mathcal{S}\left(\mathcal{H}_{A}^{\otimes n}\right)$, be a sequence of states satisfying

$$
\lim _{n \rightarrow \infty}\left\|\omega_{n}-\omega^{\otimes n}\right\|=0 .
$$

Then

$$
S(\omega) \leq \liminf _{n \rightarrow \infty} \frac{1}{n} S\left(\omega_{n}\right)
$$

Proof. Let $\pi$ be the projection operator on the support of $\omega$, and let

$$
\eta_{n}:=\pi^{\otimes n} \omega_{n} \pi^{\otimes n}, \lambda_{n}:=\left\|\eta_{n}\right\| .
$$

The trace-norm distance is non-increasing under trace-preserving completety positive maps, and in particular, under pinchings [16]. Therefore,

$$
\left\|\omega^{\otimes n}-\eta_{n}\right\|+\left(1-\lambda_{n}\right) \leq\left\|\omega^{\otimes n}-\omega_{n}\right\| .
$$

Hence, if $\lim _{n \rightarrow \infty}\left\|\omega^{\otimes n}-\omega_{n}\right\|=0$ holds, then also $\lim _{n \rightarrow \infty}\left\|\omega^{\otimes n}-\eta_{n}\right\|=0$, and $\lim _{n \rightarrow \infty} \lambda_{n}=1$. In turn, if $\lim _{n \rightarrow \infty}\left\|\omega^{\otimes n}-\eta_{n}\right\|=0$, then

$$
\lim _{n \rightarrow \infty}\left\|\lambda_{n} \omega^{\otimes n}-\eta_{n}\right\|=0
$$




$$
\left\|\lambda_{n} \omega^{\otimes n}-\eta_{n}\right\| \leq\left|\lambda_{n}-1\right|+\left\|\omega^{\otimes n}-\eta_{n}\right\| .
$$

The triangle inequality yields

$$
\begin{aligned}
\left|S\left(\omega^{\otimes n}\right)-S\left(\eta_{n}\right)\right| / n & \leq\left|S\left(\omega^{\otimes n}\right)-S\left(\eta_{n} / \lambda_{n}\right)\right| / n \\
& +\left|1-1 / \lambda_{n}\right| S\left(\eta_{n}\right) / n-\log _{2}\left(\lambda_{n}\right) /\left(n \lambda_{n}\right) .
\end{aligned}
$$

The second term on the right hand side in Eq. (22) certainly vanishes in the limit $n \rightarrow \infty$, as $S\left(\eta_{n}\right) / n \leq C$ for all $n \in \mathbb{N}$ for some appropriately chosen $C>0$. By applying Fannes' inequality [18] on the first term and by making use of Eq. (21) one can conclude that

$$
\lim _{n \rightarrow \infty} \frac{\left|S\left(\omega^{\otimes n}\right)-S\left(\pi^{\otimes n} \omega_{n} \pi^{\otimes n}\right)\right|}{n}=0
$$

if $\lim _{n \rightarrow \infty}\left\|\omega^{\otimes n}-\omega_{n}\right\|=0$. Using the function $f$ defined as $f(x)=-x \log _{2}(x)$ one finds that

$$
\liminf _{n \rightarrow \infty} \frac{1}{n} S\left(\omega_{n}\right) \geq \liminf _{n \rightarrow \infty} \frac{1}{n} \operatorname{tr}\left[\pi^{\otimes n} f\left(\omega_{n}\right)\right]=S(\omega),
$$

which is the statement of the lemma.

Proof of Proposition 4: The first step of the proof can be performed just as in Lemma 5. Let $\xi \in \mathcal{S}_{M}(\mathcal{H})$, let $\omega$ be a state that is supported on a finite-dimensional subspace of $\mathcal{S}_{M}(\mathcal{H})$, and let $\left\{\omega_{n}\right\}_{n=1}^{\infty}, \omega_{n} \in \mathcal{S}_{n M}\left(\mathcal{H}_{A}^{\otimes n}\right)$, be a sequence of states satisfying $\lim _{n \rightarrow \infty}\left\|\omega_{n}-\omega^{\otimes n}\right\|=0$. Then

$$
S(\xi|| \omega) \leq \liminf _{n \rightarrow \infty} \frac{1}{n} S\left(\xi^{\otimes n}|| \omega_{n}\right)
$$

holds. The validity of Eq. 24 can be seen as follows: the relative entropy of $\xi^{\otimes n}$ with respect to $\omega^{\otimes n}$ can be written as [17, 8

$$
\begin{aligned}
S\left(\xi^{\otimes n} \| \omega^{\otimes n}\right) & =\sup _{\mu \in[0,1]} \sup _{n} \operatorname{tr}\left[\pi _ { n } \left(f\left(\mu \xi^{\otimes n}+(1-\mu) \omega^{\otimes n}\right)\right.\right. \\
& \left.\left.-\mu f\left(\xi^{\otimes n}\right)-(1-\mu) f\left(\omega^{\otimes n}\right)\right)\right]
\end{aligned}
$$

where the supremum is taken over all finite projection operators $\pi_{n}$ on $\mathcal{H}_{A}^{\otimes n}$. So just as in Lemma 5, applying the triangle inequality and Fannes' inequality several times yields Eq. (24). In particular, one has to make use of

$$
\begin{aligned}
& \frac{\left|S\left(\mu \xi^{\otimes n}+(1-\mu) \omega^{\otimes n}\right)-S\left(\mu \xi^{\otimes n}+(1-\mu) \eta_{n}\right)\right|}{n} \\
\leq & \frac{\left|S\left(\mu \xi^{\otimes n}+(1-\mu) \omega^{\otimes n}\right)-S\left(\mu \xi^{\otimes n}+(1-\mu) \eta_{n} / \lambda_{n}\right)\right|}{n} \\
+ & \frac{\left|S\left(\mu \xi^{\otimes n}+(1-\mu) \eta_{n} / \lambda_{n}\right)-S\left(\mu \xi^{\otimes n}+(1-\mu) \eta_{n}\right)\right|}{n} .
\end{aligned}
$$


Eq. (24) is now the starting point of an argument along the line of the argument of Ref. [8]: Since the free energy can be expressed in terms of the relative entropy according to

$$
\frac{1}{n} F\left(\omega_{n}, \beta, H^{\otimes n}\right)=\left(S\left(\sigma_{\beta}^{\otimes n} \| \omega_{n}\right) / n-\log _{2}\left(\operatorname{tr}\left[e^{-\beta H}\right]\right) / \beta,\right.
$$

it has the property

$$
F(\omega, \beta, H) \leq \liminf _{n \rightarrow \infty} \frac{1}{n} F\left(\omega_{n}, \beta, H^{\otimes n}\right),
$$

implying that

$$
\beta \operatorname{tr}[\omega H]-S(\omega) \leq \liminf _{n \rightarrow \infty} \frac{1}{n}\left(\beta \operatorname{tr}\left[\omega_{n} H^{\otimes n}\right]-S\left(\omega_{n}\right)\right),
$$

and, therefore,

$$
\begin{aligned}
-S(\omega) & \leq \liminf _{n \rightarrow \infty}\left(-S\left(\omega_{n}\right) / n\right) \\
& +\beta \limsup _{n \rightarrow \infty}\left|\operatorname{tr}\left[\omega_{n} H^{\otimes n}\right]\right| / n+\beta \operatorname{tr}[\omega H]
\end{aligned}
$$

for all $\beta>0$. Again, the sum of the last two terms of Eq. (29) is bounded from above by $2 \beta M$, providing the inequality

$$
-S(\omega) \leq \liminf _{n \rightarrow \infty}\left(-S\left(\omega_{n}\right) / n\right) .
$$

Eq. (30) and the statement of Lemma 4 then imply that $S(\omega)=\lim _{n \rightarrow \infty} S\left(\omega_{n}\right)$. Finally, one can argue as in Proposition 3, by taking $\omega:=\phi_{B}(\sigma)$ and $\omega_{n}:=\Phi_{B}\left(\sigma_{n}\right)$. This gives rise to the asymptotic continuity property stated in Eq. (16).

This asymptotic continuity property concerned the entropy of entanglement. A similar property would be desirable for sequences of states that are possibly mixed states. In this context it is of interest to see whether typical measures for mixed states give, loosely speaking, the appropriate value when they are evaluated for pure states. More precisely, one may ask whether entanglement measures are trace-norm continuous in pure states, and whether they exhibit an asymptotic continuity property. We will discuss these questions for the relative entropy of entanglement [20] and the entanglement of formation [10], starting with the latter one:

For a given mixed state $\sigma \in \mathcal{S}_{M}(\mathcal{H}), M>0$, there exist (uncountably many) sequences $\left\{p^{(i)}\right\}_{i=1}^{\infty}$ of positive numbers forming a probability distribution, $p^{(1)} \geq$ $p^{(2)} \geq \ldots$, and sequences $\left\{\psi^{(i)}\right\}_{i=1}^{\infty}$ of state vectors $\psi^{(i)} \in \mathcal{H}$ such that

$$
\sigma=\sum_{i} p_{i}\left|\psi^{(i)}\right\rangle\left\langle\psi^{(i)}\right|
$$

The pair $\left(\left\{p^{(i)}\right\}_{i=1}^{\infty},\left\{\psi^{(i)}\right\}_{i=1}^{\infty}\right)$ will be called decomposition of $\sigma$. As for a finitedimensional Hilbert space one may define the entanglement of formation as

$$
E_{F}(\sigma)=\inf \sum_{i} p_{i}\left(S \circ \Phi_{B}\right)\left(\left|\psi^{(i)}\right\rangle\left\langle\psi^{(i)}\right|\right),
$$


where the infimum is understood with respect to all decompositions of $\sigma$. In the case of a finite-dimensional Hilbert space the infimum is always attained, and by virtue of Caratheodory's theorem one can find an upper bound for the required number of terms in a a decomposition of the state. It is worth noting that, using a different language, a decomposition of a state $\sigma$ can also be represented by probability measures $\mu_{\sigma}$ on state space with the barycenter $b\left(\mu_{\sigma}\right)=\sigma$. The entanglement of formation can then be defined via a minimization of the mean local von-Neumann entropy with respect to all probability measures with the same barycenter. This approach has been pursued in Ref. [4] , where the entanglement of formation has been studied in the case of a bi-partite system, with of the systems being finite-dimensional. The main difference of the situation in Ref. [ [4] and in the present paper is that in the case of one finite-dimensional subsystem the trace-norm continuity of the von-Neumann entropy is available.

Proposition 6. Let $M>0$, let $\sigma \in \mathcal{S}_{M}(\mathcal{H}), \sigma=|\psi\rangle\langle\psi|$, be a pure state, and let $\left\{\sigma_{k}\right\}_{k=1}^{\infty}$ a sequence of states $\sigma_{k} \in \mathcal{S}_{M}(\mathcal{H})$ with $\sigma_{k} \longrightarrow \sigma$ in trace-norm. Then

$$
\lim _{k \rightarrow \infty}\left|E_{F}\left(\sigma_{k}\right)-E_{F}(\sigma)\right|=0 .
$$

Proof. We start with proving the lower semi-continuity of $E_{F}$ in $\sigma$. Let $\xi>0$ be a number satisfying $E_{F}\left(\sigma_{k}\right) \leq \xi$ for all $k \in \mathbb{N}$. For all $\varepsilon>0$ there exists a decomposition $\left(\left\{p_{k}^{(i)}\right\}_{i=1}^{\infty},\left\{\psi_{k}^{(i)}\right\}_{i=1}^{\infty}\right)$ of each $\sigma_{k}$ such that

$$
\sum_{i} p_{k}^{(i)}\left(S \circ \Phi_{B}\right)\left(\left|\psi_{k}^{(i)}\right\rangle\left\langle\psi_{k}^{(i)}\right|\right) \leq \xi+\varepsilon .
$$

The fact that $\sigma_{k} \longrightarrow \sigma=|\psi\rangle\langle\psi|$ in trace-norm implies that there exists a sequence of real numbers $\left\{f_{k}\right\}_{k=1}^{\infty}$ with $\lim _{k \rightarrow \infty} f_{k}=0$, and such that

$$
\lim _{k \rightarrow \infty} \sum_{i} p_{k}^{(i)} \theta\left(f_{k}-\|\left|\psi_{k}^{(i)}\right\rangle\left\langle\psi_{k}^{(i)}\right|-\sigma \|\right)=1,
$$

where $\theta: \mathbb{R} \longrightarrow\{0,1\}$ is the Heaviside function. For each $k$ construct a sequence $\left\{q_{k}^{(i)}\right\}_{i=1}^{\infty}$ of real numbers as

$$
q_{k}^{(i)}= \begin{cases}p_{k}^{(i)}, & \text { if } f_{k}-\|\left|\psi_{k}^{(i)}\right\rangle\left\langle\psi_{k}^{(i)}\right|-\sigma \|>0, \text { and } \\ q_{k}^{(i)}=0 & \text { otherwise. }\end{cases}
$$

Similarly, define a sequence $\left\{\phi_{k}^{(i)}\right\}_{k=1}^{\infty}$ through $\phi_{k}=\psi_{k}$ if $f_{k}-\|\left|\psi_{k}^{(i)}\right\rangle\left\langle\psi_{k}^{(i)}\right|-\sigma \|>0$, and $\phi_{k}=\psi$ otherwise. It follows that

$$
\sum_{i} q_{k}^{(i)}\left(S \circ \Phi_{B}\right)\left(\left|\phi_{k}^{(i)}\right\rangle\left\langle\phi_{k}^{(i)}\right|\right) \leq \xi+\varepsilon
$$

$\lim _{k \rightarrow \infty} \|\left|\phi_{k}^{(i)}\right\rangle\left\langle\phi_{k}^{(i)}\right|-\sigma \|=0$ for all $i$, and $\lim _{k \rightarrow \infty} \sum_{i} q_{k}^{(i)}=1$. Hence, due to the lower-semicontinuity of the von-Neumann entropy we can conclude that also $(S \circ$ $\left.\Phi_{B}\right)(\sigma) \leq \xi+\varepsilon$. As $\varepsilon>0$ was arbitrary, it follows that $E_{F}(\sigma) \leq \xi$, which means that 
$E_{F}$ is lower semi-continuous in $\sigma$. Note that the constraint on the mean energy was not needed in this step.

The second part of the proof will be concerned with the upper semi-continuity in $\sigma$. Let $\xi \in \mathbb{R}$ such that $E_{F}\left(\sigma_{k}\right) \geq \xi$ for all $k \in \mathbb{N}$. Essentially, the proof goes again to a large extent along the line of the argument in Ref. [8], where additionally, we make use of the convexity of the relative entropy functional. Let $\left(\left\{p_{k}^{(i)}\right\}_{i=1}^{\infty},\left\{\psi_{k}^{(i)}\right\}_{i=1}^{\infty}\right)$ be a decomposition of $\sigma_{k}$, and let $\omega:=\Phi_{B}(\sigma), \omega_{k}:=\Phi_{B}\left(\sigma_{k}\right)$, and $\omega_{k}^{(i)}:=\Phi_{B}\left(\left|\psi_{k}^{(i)}\right\rangle\left\langle\psi_{k}^{(i)}\right|\right)$. On using both the lower semi-continuity and the convexity of the relative entropy functional one obtains

$$
S\left(\omega \| \sigma_{\beta}\right) \leq \liminf _{k \rightarrow \infty} S\left(\omega_{k} \| \sigma_{\beta}\right) \leq \liminf _{k \rightarrow \infty} \sum_{i} p_{k}^{(i)} S\left(\omega_{k}^{(i)} \| \sigma_{\beta}\right) .
$$

Therefore, $F\left(\omega, \beta, H_{A}\right) \leq \liminf _{k \rightarrow \infty} \sum_{i} p_{k}^{(i)} F\left(\omega_{k}^{(i)}, \beta, H_{A}\right)$, which means that

$$
\beta \operatorname{tr}\left[\omega H_{A}\right]-S(\omega) \leq \liminf _{k \rightarrow \infty}\left(\beta \operatorname{tr}\left[\omega_{k} H_{A}\right]-\sum_{i} p^{(i)} S\left(\omega_{k}^{(i)}\right)\right)
$$

and

$$
-S(\omega) \leq \liminf _{k \rightarrow \infty} \sum_{i} p^{(i)}\left(-S\left(\omega_{k}^{(i)}\right)\right)+\beta \limsup _{k \rightarrow \infty}\left|\operatorname{tr}\left[\omega_{k} H_{A}\right]\right|+\beta \operatorname{tr}\left[\omega H_{A}\right] .
$$

Hence, we arrive at

$$
-S(\omega) \leq \liminf _{k \rightarrow \infty} \sum_{i} p^{(i)}\left(-S\left(\omega_{k}^{(i)}\right)\right) .
$$

As $E_{F}\left(\sigma_{k}\right) \geq \xi$ for all $k \in \mathbb{N}$, and the above decomposition is not necesarily optimal for $E_{F}\left(\sigma_{k}\right)$,

$$
\sum_{i} p^{(i)}\left(S \circ \Phi_{B}\right)\left(\left|\psi_{k}^{(i)}\right\rangle\left\langle\psi_{k}^{(i)}\right|\right) \geq \xi
$$

The last step is to see that therefore, $\left(S \circ \Phi_{B}\right)(\sigma) \geq \xi$, which means that $E_{F}$ is also upper semi-continuous in $\sigma$.

Therefore, we conclude, the entanglement of formation for pure states can indeed by simply identified with the entropy of entanglement on the set $\mathcal{S}_{M}(\mathcal{H})$. A similar argument applies again on the asymptotic limit: if we have a series of mixed states that converges in trace-norm to the $n$-fold tensor product of a pure state with a finite support, then, again, one can expect an asymptotic continuity as in Proposition 4:

Proposition 7. Let $\sigma \in \mathcal{S}_{M}(\mathcal{H}), M>0$, be a pure state that is supported on a finitedimensional subspace of $\mathcal{S}(\mathcal{H})$, and let $\left\{\sigma_{n}\right\}_{n=1}^{\infty}, \sigma_{n} \in \mathcal{S}_{n M}\left(\mathcal{H}^{\otimes n}\right)$, be a sequence of states satisfying $\lim _{n \rightarrow \infty}\left\|\sigma_{n}-\sigma^{\otimes n}\right\|=0$. Then

$$
\lim _{n \rightarrow \infty} \frac{\left|E_{F}\left(\sigma^{\otimes n}\right)-E_{F}\left(\sigma_{n}\right)\right|}{n}=0 .
$$


Proof. One can proceed in the same way as Proposition 6. Instead of the mere lower-semicontinuity of the von-Neumann entropy one has to make use of the statement of Lemma 5.

For general mixed states the actual minimization that is necessary in order to evaluate the entanglement of formation in the infinite-dimensional setting appears to be quite intractable, and we do not consider the entanglement of formation in this situation.

\section{RELATIVE ENTROPY OF ENTANGLEMENT}

In the finite-dimensional setting the relative entropy of entanglement is defined as the minimal 'distance' of a given state to an appropriately chosen set that is closed under local quantum operations together with classical communication (LOCC) and which includes the identity [20]. The distance is quantified by means of the relative entropy functional. Most typically, one chooses either the set of separable states or the set of states with a positive partial transpose, the set of PPT states. In case that the Hilbert spaces are infinite-dimensional, one can define the relative entropy of entanglement in the same way - with the exception that care is needed in the definition of the notion of the set of separable states. As in Refs. [21, 3] we define the set of separable states $\mathcal{D}(\mathcal{H}) \subset \mathcal{S}(\mathcal{H})$ as the set of states for which there exists a sequence $\left\{\omega_{k}\right\}_{k=1}^{\infty}, \omega_{n} \in \mathcal{S}(\mathcal{H})$, such that $\omega_{k} \longrightarrow \omega$ in trace-norm, and such that each $\omega_{k}$ is of the form

$$
\omega_{k}=\sum_{i} p_{i}^{(n)} \eta_{A}^{(k, i)} \otimes \eta_{B}^{(k, i)}
$$

where $\eta_{A}^{(k, i)} \in \mathcal{S}\left(\mathcal{H}_{A}\right), \eta_{B}^{(k, i)} \in \mathcal{S}\left(\mathcal{H}_{B}\right)$ for all $i, k$, and $\left\{p_{i}^{(k)}\right\}_{i=1}^{\infty}$ form probability distributions for all $k \in \mathbb{N}$ : I.e., one requires that the state $\omega$ can be approximated in trace-norm by convex combinations of products [21, 3], which in turn means that $\mathcal{D}(\mathcal{H})$ is the closed convex hull of the set of products (with respect to the topology induced by the trace-norm). Another reasonable set is the set $\mathcal{P}(\mathcal{H}) \subset \mathcal{S}(\mathcal{H})$ of states that can be approximated in (trace-norm) by PPT states, that is, states $\omega \in \mathcal{S}(\mathcal{H})$ for which their partial transpose $\omega^{T_{A}}$ is again a state. The relative entropy of entanglement is the map $E_{R}: \mathcal{S}(\mathcal{H}) \longrightarrow \mathbb{R}^{+}$defined as

$$
E_{R}(\sigma)=\inf _{\rho \in \mathcal{D}(\mathcal{H})} S(\sigma \| \rho),
$$

or, alternatively, with $\mathcal{D}(\mathcal{H})$ being replaced by $\mathcal{P}(\mathcal{H})$. Again, we are interested whether the relative entropy of entanglement is trace-norm continuous in pure states. Here, we will however show the stronger property of continuity on the whole state space:

Proposition 8. The relative entropy of entanglement $E_{R}: \mathcal{S}_{M}(\mathcal{H}) \longrightarrow \mathbb{R}^{+}$is trace-norm continuous. 
Proof. Let $\left\{\sigma_{k}\right\}_{k=1}^{\infty}$ be a sequence of states $\sigma_{k} \in \mathcal{S}_{M}(\mathcal{H})$ for which $\lim _{k \rightarrow \infty} \| \sigma_{k}-$ $\sigma \|=0$. Let $\rho_{k} \in \mathcal{S}_{M}(\mathcal{H})$ for each $\sigma_{k}$ be the state for which $E_{R}\left(\sigma_{k}\right)=S\left(\sigma_{k} \| \rho_{k}\right)$ (such a state exists, due to the compactness of the set under consideration and the lower semi-continuity of the relative entropy). Then

$$
\left|E_{R}(\sigma)-E_{R}\left(\sigma_{k}\right)\right| \leq\left|S(\sigma)-S\left(\sigma_{k}\right)\right|+\left|-\operatorname{tr}\left[\sigma \log _{2}(\rho)\right]+\operatorname{tr}\left[\sigma_{k} \log _{2}\left(\rho_{k}\right)\right]\right| .
$$

As $\sigma, \sigma_{k} \in \mathcal{S}_{M}(\mathcal{H}), \lim _{n \rightarrow \infty}\left|S(\sigma)-S\left(\sigma_{k}\right)\right|=0$. The first term on the right hand side of Eq. (??) is bounded from above by

$$
\begin{aligned}
\left|-\operatorname{tr}\left[\sigma \log _{2}(\rho)\right]+\operatorname{tr}\left[\sigma_{k} \log _{2}\left(\rho_{k}\right)\right]\right| & \leq\left|-\operatorname{tr}\left[\sigma \log _{2}(\rho)\right]+\operatorname{tr}\left[\sigma \log _{2}\left(\omega_{k}\right)\right]\right| \\
& +\left|-\operatorname{tr}\left[\sigma \log _{2}\left(\omega_{k}\right)\right]+\operatorname{tr}\left[\sigma_{k} \log _{2}\left(\omega_{k}\right)\right]\right| \\
& +\left|-\operatorname{tr}\left[\sigma_{k} \log _{2}\left(\omega_{k}\right)\right]+\operatorname{tr}\left[\sigma_{k} \log _{2}\left(\rho_{k}\right)\right]\right|,
\end{aligned}
$$

where $\omega_{k}:=\left\|\sigma-\sigma_{k}\right\| \sigma_{\beta}+\left(1-\left\|\sigma-\sigma_{k}\right\|\right) \rho$. Due to the operator monotonicity of the logarithm

$$
-\operatorname{tr}\left[\sigma \log _{2}(\rho)\right]+\operatorname{tr}\left[\sigma \log _{2}\left(\omega_{k}\right)\right] \geq \log \left(1-\left\|\sigma-\sigma_{k}\right\|\right)
$$

holds. But $-\operatorname{tr}\left[\sigma \log _{2}(\rho)\right] \leq-\operatorname{tr}\left[\sigma \log _{2}\left(\omega_{k}\right)\right]$, and therefore,

$$
\lim _{k \rightarrow \infty}\left|-\operatorname{tr}\left[\sigma \log _{2}(\rho)\right]+\operatorname{tr}\left[\sigma \log _{2}\left(\omega_{k}\right)\right]\right|=0 .
$$

In the same way one finds that $\lim _{k \rightarrow \infty}\left|-\operatorname{tr}\left[\sigma_{k} \log _{2}\left(\omega_{k}\right)\right]+\operatorname{tr}\left[\sigma_{k} \log _{2}\left(\rho_{k}\right)\right]\right|=0$. The third term on the right hand side of Eq. (46) can be dealt with just as in Ref. [12], where the Gibbs state plays the role of the maximally mixed state: Since

$$
\left|-\operatorname{tr}\left[\sigma \log _{2}\left(\omega_{k}\right)\right]+\operatorname{tr}\left[\sigma_{k} \log _{2}\left(\omega_{k}\right)\right]\right| \leq\left\|\sigma-\sigma_{k}\right\|\left|\| \log _{2}\left(\omega_{k}\right)\right|||,
$$

one can again make use of the operator monotonicity of the logarithm to find

$$
\text { || } \log _{2}\left(\omega_{k}\right)||\left|\leq-\log _{2}\left(\left\|\sigma-\sigma_{k}\right\|\right)+\right||| \log _{2}\left(\sigma_{\beta}\right) \mid \|,
$$

and hence, $\lim _{k \rightarrow \infty}\left|-\operatorname{tr}\left[\sigma \log _{2}\left(\omega_{k}\right)\right]+\operatorname{tr}\left[\sigma_{k} \log _{2}\left(\omega_{k}\right)\right]\right|=0$. Collecting the partial results, one finds that $\lim _{k \rightarrow \infty}\left|E_{R}(\sigma)-E_{R}\left(\sigma_{k}\right)\right|=0$.

The corresponding asymptotic statement reads as follows:

Proposition 9. Let $\sigma \in \mathcal{S}_{M}(\mathcal{H}), M>0$, be a pure state that is supported on a finitedimensional subspace of $\mathcal{S}(\mathcal{H})$, and let $\left\{\sigma_{n}\right\}_{n=1}^{\infty}, \sigma_{n} \in \mathcal{S}_{n M}\left(\mathcal{H}^{\otimes n}\right)$, be a sequence of states satisfying $\lim _{n \rightarrow \infty}\left\|\sigma_{n}-\sigma^{\otimes n}\right\|=0$. Then

$$
\lim _{n \rightarrow \infty} \frac{\left|E_{R}\left(\sigma^{\otimes n}\right)-E_{R}\left(\sigma_{n}\right)\right|}{n}=0 .
$$

Proof. One may proceed as before. Again,

$$
\left|E_{R}\left(\sigma^{\otimes n}\right)-E_{R}\left(\sigma_{n}\right)\right| \leq\left|S\left(\sigma^{\otimes n}\right)-S\left(\sigma_{n}\right)\right|+\mid-\operatorname{tr}\left[\sigma^{\otimes n} \log _{2}\left(\eta_{n}\right)+\operatorname{tr}\left[\sigma_{n} \log _{2}\left(\rho_{n}\right)\right] \mid .\right.
$$


By means of Fannes' inequality one can infer that $\lim _{n \rightarrow \infty}\left|S\left(\sigma^{\otimes n}\right)-S\left(\sigma_{n}\right)\right| / n=0$. The second term on the right hand side can be bounded from above as in Proposition 8 , but now by making use of the $n$-fold product $\sigma_{\beta}^{\otimes n}$ of the Gibbs state $\sigma_{\beta}$.

This statement ends the considerations of entanglement measures in the infinitedimensional setting. We have seen that if the mean energy is bounded from above, and under an assumption concerning the spectrum of the Hamiltonian $H$, then several entanglement measures retain their trace-norm continuity.

\section{NON-EXISTENCE OF A SEPARABLE BALL}

Motivated by the findings of the two previous sections one might be tempted to think that with the help of a constraint on the mean energy, a separable neighbourhood of some mixed state can be recovered. In finite-dimensional bi-partite quantum systems, the situation is as follows: for any dimension of the Hilbert space of a bipartite system there exists a separable neighbourhood of the maximally mixed state - the tracial-state [3,22]: Whenever a state is closer to the maximally mixed state with respect to the trace-norm (or any other norm), then one can be sure that the state is not entangled. The size of this neighbourhood is however not independent of the dimension of the system: loosely speaking, it decreases with increasing dimension of the underlying Hilbert space. In infinite-dimensional systems, the set of entangled states is trace-norm dense in the state space of the system, and there is no separable neighbourhood of any mixed state [3]. It is the purpose of the subsequent proposition to show that also under the restriction that the mean energy is bounded from above, no such neighbourhood can be reestablished.

Proposition 10. For any $\varepsilon>0, M>0$, and $\sigma \in \mathcal{S}_{M}(\mathcal{H})$ there exists an entangled state $\rho \in \mathcal{S}_{M}(\mathcal{H})$ with the property that $\|\sigma-\rho\|<\varepsilon$.

Proof. We will prove this statement by constructing a sequence $\left\{\rho_{k}\right\}_{k=1}^{\infty}$ of entangled states $\rho_{k} \in \mathcal{S}_{M}(\mathcal{H})$ that satisfy $\rho_{k} \longrightarrow \sigma$ in trace-norm. Essentially, the idea is to construct a sequence $\left\{\rho_{k}\right\}_{k=1}^{\infty}$ of states which converge to $\sigma$ in trace norm, but which are entangled on a $2 \times 2$-dimensional subspace. Let $k \in \mathbb{N}$,

$$
\mathcal{L}_{k}:=\operatorname{span}\left\{\phi_{A}^{(i)} \otimes \phi_{B}^{(j)}: i, j \in\{k, k+1\}\right\},
$$

with $\phi_{A}^{(i)}$ and $\phi_{B}^{(j)}$ as in section 2 , and denote by $\pi_{k}$ the projection on the Hilbert space

$$
\mathcal{K}_{k}:=\operatorname{span}\left\{\phi_{A}^{(l)} \otimes \phi_{B}^{(m)}: l, m=0, \ldots, k-1\right\} .
$$

Let $\lambda_{k}:=\operatorname{tr}\left[\pi_{k} \sigma \pi_{k}\right]$. If $\lambda_{k}<1$, then one can find a state vector $\phi_{k} \in \mathcal{L}_{k}$ such that the partial transpose $\left(\left|\phi_{k}\right\rangle\left\langle\phi_{k}\right|\right)^{T_{A}}$ is not positive, and such that

$$
\operatorname{tr}[H \sigma]-\operatorname{tr}\left[H \pi_{k} \sigma \pi_{k}\right] \geq\left(1-\lambda_{k}\right) \operatorname{tr}\left[H\left|\phi_{k}\right\rangle\left\langle\phi_{k}\right|\right] .
$$

In this case set

$$
\rho_{k}:=\pi_{k} \sigma \pi_{k}+\left(1-\lambda_{k}\right)\left|\phi_{k}\right\rangle\left\langle\phi_{k}\right|
$$


If $\lambda_{k}=1$, then take $\rho_{k}:=(1-1 / k) \sigma+1 / k\left|\phi_{k}^{+}\right\rangle\left\langle\phi_{k}^{+}\right|$, where $\phi_{k}^{+}:=\left(\phi_{A}^{(k)} \otimes \phi_{B}^{(k)}+\right.$ $\left.\phi_{A}^{(k+1)} \otimes \phi_{B}^{(k+1)}\right) / \sqrt{2}$. The sequence $\left\{\rho_{k}\right\}_{k=1}^{\infty}$ has all the desired properties: by definition $\rho_{k} \longrightarrow \sigma$ in trace-norm, $\operatorname{tr}\left[H \rho_{k}\right] \leq \operatorname{tr}[H \sigma]<M$, and $\rho_{k}$ is entangled for every $k \in \mathbb{N}$ : the partial transpose of the projection of $\rho_{k}$ on $\mathcal{L}_{k}$ is not positive by construction, and hence, this projection is entangled, according to the Peres-Horodeckicriterion [23]. Therefore, $\rho_{k}$ is also an entangled state.

\section{SUMMARY AND CONCLUSION}

The content of this paper may be summarized in a nutshell as follows: if one imposes a restriction on the mean energy and requires that the Gibbs state exists, then several entanglement measures are trace-norm continuous, just as in the finitedimensional setting. We investigated the entropy of entanglement, the entanglement of formation, and the relative entropy of entanglement, both for a single copy and in the asymptotic limit. The findings are in a sense of less practical than of reassuring nature: if the energy is bounded, then one may truncate the state with respect to a finite dimensional subspace and evaluate the degree of entanglement on this subspace. This could be relevant in the situation where one considers a quantum operation that acts on a finite-dimensional subspace of a quantum system, which has nevertheless an infinite-dimensional Hilbert space. An example of this type is the state manipulation of the center-of-mass mode of ions in an ion trap, where one considers only certain excitations. In an imperfect implementation of the quantum operation the resulting state may not be strictly confined to a finite-dimensional subset any more. But if the difference of the actual state and the restriction on the finite-dimensional subset is very small, one should not expect to have a completely different situation as far as the entanglement of the state is concerned, by taking the rest of the Hilbert space. In this sense are the findings also relevant for investigations of the degree of entanglement of Gaussian quantum states. Needless to say, there are many issues that remain to be addressed. For example, the concepts of entanglement of distillation, the 'one-shot'-distillation involving a single copy only, and the asymptotic entanglement cost have so far only been formulated in the finite dimensional case in a rigorous manner, and await a systematic investigation for infinite-dimensional quantum systems.

\section{ACKNOWLEDGEMENTS}

We would like to thank K. Życzkowski, R.F. Werner and J.I. Cirac for valuable remarks. This work has been supported by the European Union (EQUIP - IST-199911053), the Alexander-von-Humboldt-Foundation, the ESF, and the EPSRC. 
[1] D. Bohm, Quantum Theory (Prentice-Hall, Englewood Cliffs, NJ, 1951).

[2] R.F. Werner, Quantum information - an introduction to basic theoretical concepts and experiments, in Springer Tracts in Modern Physics 173 (Springer, Heidelberg, 2001); M.B. Plenio and V. Vedral, Contemp. Phys. 39, 431 (1998); M. Lewenstein, D. Bruß, J. I. Cirac, B. Kraus, M. Kus, J. Samsonowicz, A. Sanpera, and R. Tarrach, J. Mod. Opt. 47, 2481 (2000); M. Horodecki, Quant. Inf. Comp. 1, 3 (2001); M.A. Nielsen and I.L. Chuang, Quantum Computation and Quantum Information (Cambridge University Press, Cambridge, 2000).

[3] R. Clifton and H. Halvorson, Phys. Rev. A 61, 012108 (2000); H. Halvorson and R. Clifton, J. Math. Phys. 41, 1711 (2000); R. Clifton, H. Halvorson, and A. Kent, Phys. Rev. A 61, 042101 (2000); T. Bröcker and R.F. Werner, J. Math. Phys. 36, 62 (1995); P. Horodecki and M. Lewenstein, Phys. Rev. Lett. 85, 2657 (2000); P. Horodecki, J.I. Cirac, and M. Lewenstein, quant-ph/0103076.

[4] A.W. Majewski, J. Phys. A 35, 123 (2002).

[5] R. Simon, Phys. Rev. Lett. 84, 2726 (2000); R.F. Werner and M.M. Wolf, Phys. Rev. Lett. 86, 3658 (2001); G. Giedke, B. Kraus, M. Lewenstein, J.I. Cirac, Phys. Rev. Lett. 87, 167904 (2001); G. Giedke, L.-M. Duan, J.I. Cirac, P. Zoller, Quant. Inf. Comp. 1, 79 (2001).

[6] J. Eisert and M.B. Plenio, quant-ph/0109126; J. Eisert and M.B. Plenio, quant-ph/0111016.

[7] S. Lloyd and S.L. Braunstein, Phys. Rev. Lett. 80, 4084 (1998); N.J. Cerf, A. Ipe, and X. Rottenberg, Phys. Rev. Lett. 85, 1754 (2000); Ch. Silberhorn, P.K. Lam, O. Weiss, F. Koenig, N. Korolkova, and G. Leuchs, Phys. Rev. Lett. 86, 4267 (2001); S. Parker, S. Bose and M.B. Plenio, Phys. Rev. A 61, 32305 (2000).

[8] A. Wehrl, Rev. Mod. Phys. 50, 221 (1978); A. Wehrl, Rep. Math. Phys. 10, 159 (1976).

[9] A similar observation has been made in D. Wojcik, I. Bialynicki-Birula, and K. Życzkowski, Phys. Rev. Lett. 85, 5022 (2000), where the existence of fractal states in Hilbert space has been demonstrated. It had also been shown that fractal states do not exist if one introduces an energy constraint.

[10] C.H. Bennett, D.P. DiVincenzo, J.A. Smolin, and W.K. Wootters, Phys. Rev. A 54, 3824 (1996).

[11] P. Hayden, M. Horodecki, and B.M. Terhal, quant-ph/0008134

[12] M.J. Donald and M. Horodecki, Phys. Lett. A 264, 257 (1999).

[13] K. Audenaert, J. Eisert, E. Jane, M.B. Plenio, S. Virmani, and B. de Moor, Phys. Rev. Lett. 87, 217902 (2001).

[14] M.J. Donald, M. Horodecki, and O. Rudolph, quant-ph/0105017.

[15] In the finite dimensional setting the asymptotic continuity in this sense - together with convexity, normalization, and monotonicity under local operations - is required in order to specify the entropy of entanglement as the unique measure of entanglement [14].

[16] R. Bhatia, Matrix Analysis (Springer, Heidelberg, 1997).

[17] M. Ohya and D. Petz, Quantum Entropy and Its Use (Springer, Heidelberg, 1993).

[18] Let $\sigma$ and $\rho$ be states on a finite dimensional Hilbert space $\mathcal{H}$ with dimension $d$. Fannes' inequality [19,17] states that if $\|\sigma-\rho\|<1 / 3$, then

$$
|S(\sigma)-S(\rho)| \leq \log (d)\|\sigma-\rho\|-\|\sigma-\rho\| \log (\|\sigma-\rho\|) .
$$

[19] M. Fannes, Commun. Math. Phys. 31, 291 (1973).

[20] V. Vedral, M.B. Plenio, M.A. Rippin, and P.L. Knight, Phys. Rev. Lett. 78, 2275 (1997); V. Vedral and M.B. Plenio, Phys. Rev. A 57, 1619 (1998).

[21] R.F. Werner, Phys. Rev. A 40, 4277 (1989).

[22] A.O. Pittenger and M.H. Rubin, Phys. Rev. A 62, 2313 (2000); G. Vidal and R. Tarrach, Phys. Rev. A 59, 141 (1999); K. Zyczkowski, P. Horodecki, A. Sanpera and M. Lewenstein, Phys. Rev. A 58, 883 (1998).

[23] A. Peres, Phys. Rev. Lett. 77, 1413 (1996); P. Horodecki, Phys. Lett. A 232, 333 (1997). 